\title{
The Evolution, Appreciation and Representation of Music
}

\author{
Mamatha Bhat*, B.Sc., Sharmila Udupa
}

\section{INTRODUCTION}

The playing and singing of music has a profound effect on everyone, whatever the style of music to which one listens. What is miraculous is the complexity of music production by the performer, and how it can reach across to the listener and evoke certain emotions. Music can bring images to mind, trigger reminiscences of times past, and bring us to our feet. Music is a blend of melody, rhythm and harmony, and rendering it requires a great deal of concentration and memory power. It is through immense coordination that these intricate patterns can be produced; the underlying neural mechanisms are most definitely complex. These mechanisms bring about the question, "how is a multifaceted task such as music represented in the brain, and how does this representation stimulate the actions necessary for music production?"

The arrival of novel neuroimaging techniques such as fMRI (functional magnetic resonance imaging) and PET (positron emission tomography) has enabled us to visualize which areas of the brain are stimulated under certain conditions. Researchers are using these techniques to satisfy their curiosity about brain functioning with respect to specific functions such as tasting, smelling, reading, and listening. The etiology of certain psychiatric or neurological conditions is also better understood using these methods, as they offer a view of brain stimulation in real time. It is in this context that researchers have been investigating musical representation in the brain.

The therapeutic benefits of music have only recently been realized. Music therapy is an aspect of alternative

\footnotetext{
* To whom correspondence should be addressed:

Mamatha Bhat

3655 Promenade Sir William Osler, Montreal, QC, H3G 1 Y6.

E-mail: mbhat1@po-box.mcgill.ca
}

mind-body medicine that is gradually gaining acceptance among medical professionals (2). It is known to have a soothing psychosomatic effect on terminally ill patients ( 3 ) and reduce anxiety associated with the diagnosis and treatment of cancer $(4,5)$. Improvements in the pain of cancer in patients following music therapy have been noted (6), and research into this effect has implied the music may be beneficial in lowering anxiety levels associated with cancer and its treatment (7). Thus, these studies suggest music therapy to be an effective holistic practice for palliative care. In one study, quality of life was higher for those subjects receiving music therapy, and their quality of life increased over time as they received more music therapy sessions; however, life expectancy did not differ between the group receiving music therapy and those without intervention (8).

Music has also been tried as an "analgesic" during procedures such as bronchoscopy (9) and colonoscopy (10), as it has a distracting effect. Wang et al. showed a decrease in preoperative anxiety and acute postoperative pain with the use of music therapy (11). Music therapy as a clinical intervention has been demonstrated to improve mood states with a variety of populations (12). But how is it that music can effect such change in one's state of mind?

Functional MRIs and PET scanning have afforded researchers a view of the brain regions that light up in response to musical stimulation. The processing of sounds in general has been known as a function of the Heschl's gyrus, a component of the temporal lobe. Since music involves a variety of aspects, such as melody, rhythm, harmony and fine motor coordination, its representation is indisputably more complex. This review article attempts to explore the various studies that have been accomplished in 
recent years with respect to the brain's representation of these various facets of music. In addition, it looks at the purpose of such musical representation in the brain within the context of human evolution.

\section{MUSIC APPRECIATION AS AN INNATE QUALITY}

Due to their similarities, there has been widespread speculation that music and language are processed in much the same manner (13). For example, both are systematic, rule-based, and have an infinite range of possibilities. Notably, both are audibly (and in the case of language, visually) discernable at varying degrees of transformation such as changes in tempo, pitch, and speaker/singer. Also, some studies support the claim that language and music perception are innate qualities in human beings $(14,15,16)$, with one study even relating linguistic ability to genetics (17). A prelinguistic child's capacity for musical perception is equal to that of an experienced listener. Infants recognize the familiarity of melodies across pitch and tempo changes but are not as able to detect melodies that violate the rules of musical organization (13). Also, infants below the age of six months pay more attention to sequences of consonant intervals than to those of dissonant intervals, as will be further discussed. Finally, there are indications that prenatal and/or postnatal exposure to music has no effect on the sensitivity to musical discernment, and several studies even reveal infants to be fully capable of perceiving music of any genre (13). These skills correlating to mature musical perception strongly favor the idea that appreciation of music is an innate quality.

This incredible capacity for music perception at such a tender age might find its origins in the parent-infant relationship. Lullabies and children's melodies are sung to infants at a very early age, and from the moment of birth they are spoken to in singsong voices. The genre of music that the child listens to shares a number of features with children's songs from different cultures. During the neonatal period, infants prefer renditions of a song in the maternal style to a non-maternal version of the same song by the same performer. These early social influences on music reveal the intimate relationship of social relations and musical perception. Trehub (13) speculates that it is the social nature of music and its link to positive emotional states that has permitted its growth into an elaborate system.

Hauser and McDermott believe a parallel knowledge exists within music, and it is that which contributes to its intrinsic quality (18). They have primarily used animal research so as to avoid any potential prior exposure of the subject to the music. Notably, whatever response is evoked in the animals from listening to music would reflect an auditory capacity rather than an adaptive function. Rhesus monkeys, close to humans in terms of evolution, have displayed in past studies an intrinsic inclination to tonal melodies and a similar neural response as humans to dissonant chords. It is important to bear in mind that sensitivity to musical key structure is not consistent among different species, but is within species (such as primates, infants, and adults), and that behavioral expressions may not parallel neural mechanisms at all times.

\section{EVOLUTION OF MUSIC}

If music is indeed an innate quality, the next step would be to examine its evolutionary origins. Hauser and McDermott (18) offer a number of plausible suggestions on the development of music from its origins, all heavily influenced by Noam Chomsky's (19) concept of an innate "knowledge of language". This concept refers to an unconscious set of guidelines that define grammatical judgments, comprehension, and production.

As for its purpose, some theorists state music to have developed as a sexually selective system devised to attract mates and signal mating potential, or to more generally express emotional states $(20,21)$. Other theorists believe music serves to guide and facilitate collaboration among groups of people (22). There exists yet another group of scientists who believe music does not have an adaptive source, and is simply an incidental consequence of unrelated perceptual and cognitive functions (23). Certain musical forms such as Indian classical music and gospel music are devotional and spiritually inclined. Among these possibilities, the one that remains prevalent in our social context is our usage of music as a means of expression. Human and nonhuman animals still manifest their emotional states via variable vocalizations, which show that music and emotional articulation have likely developed simultaneously (18).

Rhythmic discrimination is an auditory mechanism that seems to have developed over the course of evolution as well (24). Studies have shown that human infants and monkeys recognize differences in speech rhythms between two languages, thus suggesting that certain tapping domain-general auditory mechanisms may have evolved prior to human production of music as we know it $(24,25,26)$.

Solid evidence of a biological basis for music comes in the form of a study by Blood and Zatorre (27). They uncovered fascinating evidence as to why music is so appealing to all. PET was used to determine the neural stimulation that forms the basis of our pleasant emotional response to music. Subjects reported chills when listening to their favorite pieces of music. This 
musical euphoria was accompanied by changes in heart rate and respiration.. Cerebral blood flow increased to brain regions implicated in reward, motivation, arousal and emotion. These areas include the amygdala, the ventral striatum, the midbrain, and the structures that are activated in response to other pleasure-inducing stimuli, such as food, sex and drug abuse. This common pattern of brain circuitry, which is involved in pleasure and reward, establishes that music is linked to "biologically-relevant, survival-related stimuli"(27).

\section{MUSICAL REPRESENTATION IN THE BRAIN}

Since music processing is clearly a mental exercise, it is logical to assume that there exists a series of neural circuits involved in its perception. What is more tantalizing, however, is to think of an entire modality for its purpose. Peretz and Coltheart (28) use Fodor's (29) definition of a modality as an entity with rapid operation, automaticity, domain-specificity, informational encapsulation, neural specificity and innateness. Although these properties are more typical than necessary, information processing and domain-specificity take importance in the definition of a mental module. Using neurologically disabled individuals with specific and isolated music-related impairments (various forms of amusia), Peretz and Coltheart have provided a comprehensive and functional model strictly for music ciphering, applying the characteristics of modular organization. In their model, a neurological defect could either interfere with information flow or with a processing component, as witnessed in detailed examination of brain-damaged patients with selective defects in music processing. The model comprises over ten processing components: tonal encoding (the knowledge of scale tones within a central tone), interval analysis, and contour analysis (pitch direction between neighboring tones) all relate to pitch organization. The remaining include rhythm analysis (fragmentation of music into temporal units based exclusively on duration value), and meter analysis (extraction of an underlying temporal rhythm with reference to strong and weak beats), which deal with temporal organization; emotional expression analysis (recognition and experience of emotion within the music), musical lexicon (bank of musical lyric exposure), vocal plan formation (results in singing), associative memories (any related nonmusical information), and two more relating primarily to speech (Figure 1).

This model is unique and more inclusive than its predecessors primarily because of its extensive organization and because of its outlook on input and output. It defines input as any acoustic stimulus that can be attributed to a single source. This input then goes to

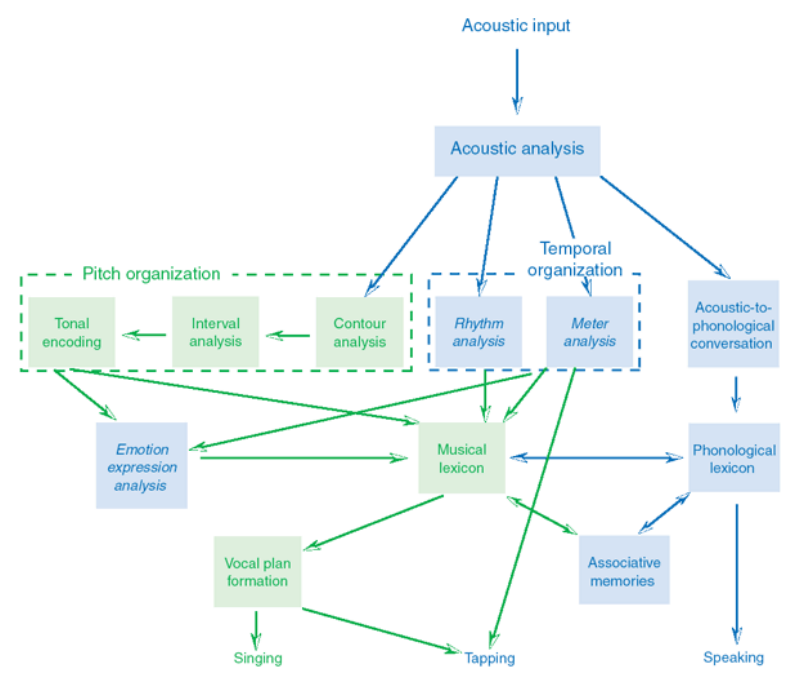

Figure 1. Modular model of music processing proposed by Peretz and Coltheart (21)

all auditory domains (not just that of music). The perceptual modules feed into an emotional analysis component, unique for each individual. Past experiences will shape what is considered emotionally appealing. The final output of the musical circuit will depend on which modality domain optimally responded to the stimulus.

\section{A) Musical Aptitude}

Perception of music has traditionally been thought of as a capacity specific to the right brain hemisphere, although a study of brain-damaged subjects by Lechevalier (30) challenged this notion. The identification and recognition of a musical piece seems to involve both hemispheres. In general, past studies have shown the left hemisphere as particularly engaged in rhythm and musical semantic representations, and the right hemisphere as specialized in melodic perception and timbre.

A study by Butcher (31) showed that auditory processing is quite different in professional musicians versus non-musicians. Primary source activity was especially localized to the anteromedial portion of Heschl's gyrus. This structure is found in the temporal lobe, and is the portion of the brain responsible for the processing of sounds. Butcher's study also detected a $130 \%$ increase in the volume of gray matter of Heschl's gyrus in professional musicians as compared to nonmusicians.

A study by Hutchinson et al. discovered that the absolute cerebellar volume of musicians was significantly greater $(p<0.001)$ than that of nonmusicians, a reflection of the highly developed and specialized motor skills they have developed early in 
life in order to play their instruments (32). The cerebellar volume increased with reported intensity of practice, i.e. the number of hours practiced.

\section{B) Pitch}

The pitch of a note is an indication of how high or low it is in the frequency range (33). The primary auditory cortex (left temporal superior gyrus of Heschl) reveals a tonotopic distribution, the anterior and lateral portions being activated for low-pitched sounds, and more medial and posterior areas being activated for high-pitched sounds (34).

Many people with musical training have the ability to quickly identify the precise position of a note in the scale without reference to any other note; this ability is known as absolute pitch. This cognitive ability seems to develop due to the interaction of genes and environmental exposure to music during childhood in particular. Such ability is seen in musicians of all traditions, due to auditory imagery and sensorimotor response codes. According to a study by Gregerson, which points toward a strong heritable component for absolute pitch, people of Eastern Asian descent have a significantly greater incidence of absolute pitch ability than those from other backgrounds (35).

When the pitch of a song changes, typical listeners show an electrophysiological response in an area of the right frontal cortex as a reflection of their on-line memory system having been reset; however, those with absolute pitch ability show no such neural activity. Rather, those with absolute pitch show activity in the posterior dorsolateral cortex when they listen to tones (36). It is likely that this is how these people label pitches to sounds readily, since this region of the brain serves an associative function.

\section{C) Harmony}

Harmony is the sound created by simultaneously occurring pitches (33). The concept of harmony is particular to Western classical music; Eastern classical music styles tend to lay emphasis on melody. With respect to harmony, studies have shown that humans are naturally more attuned to consonance than dissonance (37). Subjectively, consonance refers to two or more sound frequencies occurring simultaneously and being pleasing to the ears of the listener. In terms of semitones (a semitone being the distance between each consecutive note on the scale), consonance tends to occur when the interval size is the octave (a difference of 12 semitones), the fifth ( 7 semitones), or the major third. Objectively, it has been found that consonance is translated into pairs of notes where harmonics are integer multiples of the fundamental frequency or where the fundamental notes are expressible as a ratio of small whole numbers (2:1, 3:2, 4:3, and so on).

Adult listeners have rated the minor second (a difference of 1 semitone) as being the most dissonant sound. What is striking is that, in a study by Zentner et al., infants who were exposed to consonant sounds demonstrated visual fixation at the source of sound and significantly reduced their motor (37). When the sound was dissonant, the babies were more likely to cry and turn away from the music source, thus supporting the argument for a natural human inclination towards consonance.

Harmony has been found to localize to the right lingual gyrus and the left inferior parietal lobule (38). The activation of these areas is significantly greater in musicians.

\section{D) Melody}

Melody refers to the sequence of pitches. The superior temporal gyrus has been associated with melodic processing in many neuroimaging studies $(38,39,40)$. One study employed subjects who had undergone unilateral temporal cortectomy to relieve symptoms of epilepsy (38). This enabled determination of the roles of the various temporal lobe areas in musical processing. It was recognized that a right temporal cortectomy impaired the use of contour and interval information in melody discrimination, whereas a left temporal cortectomy adversely affected only the use of interval information. When the posterior part of the superior temporal gyrus (a portion of the auditory area) was excised, the processing of pitch and temporal variation was hindered.

In a study by Schmithorst et al. (38), unharmonized melodies bilaterally activated the superior temporal gyrus to an extent significantly greater than that associated with random tones. During this comparative exercise, musicians had significantly greater activation in the inferior parietal lobules and superior frontal gyrus bilaterally. Harmonized melodies stimulated a different activation pattern when compared to unharmonized melodies. The former generated a significantly greater activation in the right lingual gyrus and left inferior parietal lobule. A few other regions such as the right fusiform gyrus, left medial occipital gyrus, left frontal gyrus and anterior cingulate gyrus also lit up when the subjects heard harmonized melodies.

The semantic familiarity with melodies was reflected in the increased activation of the left inferior frontal and superior temporal gyri in musicians. The exact role of the parietal areas in musical processing is unknown; however auditory working memory and visuo-auditory integration are thought to be the main musical functions served. It is thought by some researchers that the supramarginal gyrus in the parietal lobe is used for 
working memory during melodic processing, while visuospatial processing of harmonies is performed by the angular gyrus. The familiarity of tunes has been traced to areas of right auditory association cortex, together with right and left frontal cortices (41). It would seem that these areas are involved in imagery for familiar tunes, as evidenced by this PET scanning study. This study by Halpern and Zatorre showed retrieval from musical semantic memory as being mediated by structures in the right frontal lobe, which conflicts with results from previous studies associating left frontal areas with all semantic retrieval. The supplementary motor area (SMA) seems to be implicated specifically in image generation, and would seem to provide the link between musical perception and action.

Cases of amelodia have been reported in the past. Such isolated disorders of music perception have given researchers clues as to the localization of musical components in the brain. For instance, a highly trained musician suffered an ischemic injury of the right temporal lobe, which impaired his ability to identify the melodies of popular music pieces. He was also unable to identify instruments being played. Thus, the right temporal lobe is critical in the perception of melody. Incidentally, this region is possibly responsible for "decoding environmental sounds, discerning emotional prosody, and identifying voices" (42).

\section{E) Rhythm}

The rhythm of music is produced by the arrangement of notes and silences of varying duration. Metre is the fundamental component of rhythm, and is represented in the anterior part of the superior temporal gyrus (43). Playing music requires the ability to maintain an internal tempo. Such maintenance of tempo is facilitated when the sequences of rhythm interval durations occur in ratios of simple integers (1:2 or 1:3). These are easier to assimilate and reproduce than rhythmic sequences with more complex meters. It is thought that such simple ratio rhythms induce internal clocks or neural oscillators that assist in the perception and production of these meters. The basal ganglia and the cerebellum are believed to play a pivotal role in timekeeping mechanisms.

Sequencing behavior is key in the production of music (43). The early phase of sequence learning in music involves linking individual sequential units one at a time. As musical training progresses, the learner starts to group elements into larger combinations or "chunks". These higher-order programs become hierarchically structured into a regular pattern of sub-sequences once the subject has become familiar with a piece.

Learning a sequence that is structurally complex places cognitive demands on the brain. It is not just a matter of rote memorization. Such learning involves executive processes, chiefly error monitoring and motor program structuring. The timing aspects of both perceptual and motor tasks have been found to activate regions such as the cerebellum, supplementary motor area (SMA), premotor cortex, basal ganglia and the parietal cortex. These areas of the brain are richly interconnected and form a circuit in the perceptionaction cycles of music production.

\section{CONCLUSION}

Music is a sensory phenomenon that elicits perceptual and emotional responses in both the performer and the spectator. The healing powers of music have come to light, and are slowly being integrated into patient care. The ability to appreciate music has evolved over the ages. What is striking are the commonalities found between perception of speech and music with respect to rhythm and tone. In a sense, one could say that musical ability overlaps with the skills involved in language and mathematics. Numerous recent studies have tried to look at human representation of music in the brain. The neural circuits involved in music are complex, and we have only begun to scratch at the surface of its phenomenal intricacies. The various aspects of music map to different areas of the brain: the semantic familiarity of melody maps to the superior temporal gyri, harmony to the right lingual gyrus, rhythm to the cerebellum and basal ganglia. Musical training seems to result in a different brain representation of music, involving a greater number of brain regions and more complex circuits. Additional research is required to be able to pinpoint the precise cortical areas recruited by the different components of music. Modular models of music processing, such as that proposed by Peretz and Coltheart, should be investigated. It is fascinating how various music components come together, and further study should be initiated to understand how this multifaceted art form is produced in such a coordinated fashion. With respect to accomplished musicians, the question arises whether their exceptional neural circuitry is already established at an early age, prior to the molding effect of musical training. A further avenue for research would be exploration of the neural circuits that fuel creativity in music. Musical forms such as jazz and Indian classical music have improvisation within a particular framework as an integral part of their rendition. Such music most definitely would result in brain activation patterns that differ from those specific to music that is played by rote memory. Music appreciation is inherent in each one of us; it is in medicine's best interest to integrate it as part of a holistic approach to the chronic care of patients. 


\section{REFERENCES}

1. Dillon WP. Neuroimaging in neurologic disorders. In: Harrison's Principles of Internal Medicine 15th edition. Toronto: McGraw-Hill Publishers; 2001: 2337

2. Fugh-Berman A. Alternative Medicine. In: Harrison's Principles of Internal Medicine 15th edition. Toronto: McGraw-Hill Publishers; 2001: 50

3. Abrams A. Music, cancer, and immunity. Clin J Oncol Nurs 2001; 5: 222-4

4. Vickers AJ, Cassileth BR. Unconventional therapies for cancer and cancer-related symptoms. The Lancet Oncology 2001; 2(4)

5. Beck SL. The therapeutic use of music for cancer-related pain. Oncol Nurs Forum 1991; 18: 1327-37.

6. Bailey LM. The effects of live music versus tape-recorded music on hospitalized cancer patients. Music Ther 1983; 3:17-28.

7. O'Kelly J. Music therapy in palliative care: current perspectives. Int J Palliat Nurs 2002; 8: 130-6

8. Hilliard RE. The effects of music therapy on the quality and length of life of people diagnosed with terminal cancer. J Music Ther 2003; 40: 113-37

9. Diette GB: Distraction therapy with nature sights and sounds reduces pain during flexible bronchoscopy: a complementary approach to routine analgesia. Chest 2003; 123(3): 941-8

10. Lee DW. Relaxation music decreases the dose of patientcontrolled sedation during colonoscopy: a prospective randomized controlled trial. Gastrointest Endosc 2002; 55(1): 33-6

11. Wang SM, Kulkarni L, Dolev J, Kain ZN. Music and preoperative anxiety: a randomized, controlled study. Anesth Analg 2002; 94(6): 1489-94

12. Magee WL, Davidson JW. The effect of music therapy on mood states in neurological patients: a pilot study. J Music Ther 2002; 39(1): 20-9

13. Trehub, Sandra E. The developmental origins of musicality. Nature Neuroscience. 2003 . 6: 669-673

14. Nowak, M.A., N.L. Komarova, and P. Niyogi. Computational and evolutionary aspects of language. Nature 2002: 417; 611617.

15. Mumby, P.J., J.R.M. Chisholm, C.D. Clark, J.D. Hedley, and J. Jaubert. Language rhythms in baby hand movements. Nature 2001: 413; 35-36.

16. Patel, A.D. Syntactic processing in language and music: different cognitive operations, similar neural resources? Music Percept. 1998: 16; 27-42.

17. Lai, C.S.L., S.E. Fisher, J.A. Hurst, F. Vargha-Khadem and A.P. Monaco. A forkhead-domain gene is mutated in a severe speech and language disorder. Nature 2001: 413; 519 - 523.

18. Hauser MD, McDermott J. The evolution of the music faculty: a comparative perspective. Nature neuroscience 2003; 6: 663-68.

19. Chomsky N. Knowledge of language: its nature, origin and use. (Praeger, New York, 1986)

20. Darwin, C. The Descent of Man and Selection in Relation to Sex (John Murray, London, 1871).

21. Miller, G.F. The Mating Mind (Doubleday, New York, 2000).

22. Hagen, E.H. \& Bryant, G.A. Music and dance as a coalition signaling system. Hum. Nat. (in press).

23. Pinker, S. How the Mind Works (Norton, New York, 1997).
24. deCharms, R.C., Blake, D.T. \& Merzenich, M.M. Optimizing sound features for cortical neurons. Science 280, 1439-1443

25. Jusczyk, P. The Discovery of Spoken Language (MIT Press, Cambridge, 1997).

26. Mehler, J. et al. A precursor of language acquisition in young infants. Cognition 29, 143-178 (1988).

27. Blood AJ and Zatorre RJ: Intensely pleasurable responses to music correlate with activity in brain regions implicated in reward and emotion. PNAS 2001; 98 (20): 11818-11823

28. Peretz I, Coltheart M. Modularity of music processing. Nature Neuroscience. 6: 688-691, 2003.

29. Fodor J. The Modularity of Mind (MIT press, Cambridge, Massachussetts, 1983).

30. Lechevalier B, Eustache F, Rossa Y: Les troubles de la perception de la musique d'origine neurologique. Paris: Masson, 1985.

31. Butcher J. Heschl's gyrus determines musical aptitude. Lancet Neurol 1(4): 207, 2002.

32. Hutchinson S, Lee LH-L, Gaab N, Schlaug G: Cerebellar Volume in Musicians http://www.musicianbrain.com/papers/ Hutchinson_Cer_CerebralC.pdf

33. Appendix F: Glossary http://www.bced.gov.bc.ca/irp/music810/ apf.htm

34. Zatorre RJ, Perry DW, Beckett CA, Westbury CF, Evans AC. Functional anatomy of musical processing listeners with absolute pitch and relative pitch. Proc. Natl. Acad. Sci USA 1998; 3172-77.

35. Gregersen P, Kowalsky E, Kohn N, Marvin E. Early childhood music education and predisposition to absolute pitch: teasing apart genes and environment. Am. J. Med. Genet. 2000; 98:28082

36. Zatorre RJ. Absolute pitch: a model for understanding the influence of genes and development on neural and cognitive function. Nature neuroscience 2003; 6:692-95

37. Zentner MR, Kagan J. Infants' perception of consonance and dissonance in music. Infant behavior \& development 1998; 21:483-92

38. Schmithorst VJ, Holland SK. The effect of musical training on music processing: a functional magnetic resonance imaging study in humans. Neuroscience Letters. 348(2):65-8, 2003 Sep 11.

39. Liegeois-Chauvel C, Peretz I, Babai M, Laguitton V, Chauvel P. Contribution of different cortical areas in the temporal lobes to music processing. Brain 1998; 121:1853-67

40. Platel H, Price C, Baron J-C, Wise R, Lambert J, Frackowiak RSJ, Lechevalier B, Eustache F. The structural components of music perception, a functional anatomical study. Brain 1997; 120: $229-243$

41. Halpern A, Zatorre RJ. When That Tune Runs Through Your Head: A PET Investigation of Auditory Imagery for Familiar Melodies. Cerebral Cortex, 9: 697-704, 1999

42. Sparr SA: Receptive amelodia in a trained musician Neurology 2002; 59(10): 1659

43. Janata P and Grafton ST. Swinging in the brain: shared neural substrates for behaviors related to sequencing and music. Nat Neurosci 2003; 6:682-87.

Mamatha Bhat is a 3rd year medical student at McGill University with a B. Sc. in Microbiology and Immunology who enjoys singing and playing Indian classical music on the violin in her spare time.

Sharmila Udupa is a 1st year medical student at McGill University with an avid interest in Indian music and performing Indian classical dance. 